\title{
Especie nueva de Haliotrema Johnston y Tiegs, 1922 (Monogenea: Dactylogyridae) de las branquias de Lactophrys polygonia (Pisces: Ostracidae) de la Bahía de Mochima, Venezuela
}

\section{New species of Haliotrema Johnston et Tiegs, 1922 (Monogenea: Dactylogyridae) of the gills of Lactophrys polygonia (Pisces: Ostracidae) of Mochima Bay, Venezuela}

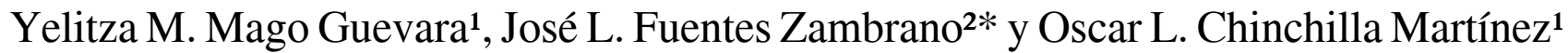

${ }^{1}$ Departamento de Biología, Escuela de Ciencias, Universidad de Oriente, Núcleo de Sucre, Cumaná, Venezuela.

${ }^{2}$ Centro Regional de Investigaciones Ambientales (CRIA), Núcleo de Nueva Esparta, Universidad de Oriente (Apartado Postal $N^{\circ}$ 147). Guatamare, Isla de Margarita, Venezuela.

*Correspondencia:fuentes@ne.udo.edu.ve

\begin{abstract}
Resumen. Se describe una especie nueva de Haliotrema (Monogenea: Dactylogyridae) de las branquias del torito Lactophrys polygonia (Ostracidae), capturados en la Bahía de Mochima, al noreste de la costas de Venezuela. Prevalencia: 77.70\%, intensidad: 70-100 e' intensidad media: 85.80. Algunas de las características que permiten separar ésta de las otras especies del género Haliotrema, son: la estructura del complejo anclas/barras, el número de células prostáticas, la estructura y posición del complejo prostático, la forma y estructura de la vagina, la condición lobulada del testículo y especialmente la estructura del complejo copulador. Este complejo está constituido por un órgano copulador tubular, bipartido, con una pieza accesoria en forma de vela con el margen exterior engrosado, que recorre en espiral toda la extensión de la fracción distal de este órgano.
\end{abstract}

Palabras clave: Taxonomía, Monogenea, peces.

\begin{abstract}
A new species of Haliotrema (Monogenea: Dactylogyridae) is described from the gills of the «torito» Lactoprhys polygonia (Ostraciodae), captured in Mochima Bay, northeast of the coast of Venezuela. Prevalence: 77.70\%; intensity: 70-100; mean intensity: 85.80. Some of the characteristics that distinguish the new species are: the structure of the anchor/bar complex, the number of prostatic glands, the structure and position of the prostatic reservoir, the form and structure of the vagina, the presence of lobulate testicle, and especially the structure of the copulatory complex. The latter is formed by a tubular, bipartite copulatory organ, with a sail-shaped accessory piece with engrossed external margin, forming a spiral running all along the distal portion.
\end{abstract}

Key words: Taxonomy, Monogenea, fishes.

\section{Introducción}

El género Haliotrema pertenece a la familia Dactylogyridae Bychowsky, 1933 e incluye 148 especies descritas en peces pertenecientes a 33 familias de teleosteos (Kritsky y Stephans, 2001). Kritsky y Boeger (2002) excluyen de este número a $H$. lutiani, $H$. fastigatum, $H$. longibaculum, $H$. paracanthi, H. torquecirrus, $H$. tubocirrus, $H$. bychowskyi y $H$. chrysotaeniae, todas ellas halladas en lutjánidos, y las transfieren al género Euryhaliotrema Kritsky y Boeger, 2002, cuya característica más distintiva es la presencia de órgano copulatorio con base bulbosa. Yamaguti (1963) reconoció un total de quince especies, posteriormente muchos son los trabajos que han incorporado este género especies y combinaciones nuevas, entre los cuales destacan Yamaguti (1968) quien describió 35 especies nuevas en teleósteos de Hawaii; Young (1968) aportó la descripción de 22 especies nuevas y realizó nuevas combinaciones para hospederos de diversas localidades geográficas; Bychowsky y Nagibina (1970) refieren 15 especies nuevas al sur de China y sugieren tres combinaciones nuevas en peces del Japón; Zhukov (1976, 1980a, 1980b, 1981) registró un total de 27 especies nuevas, encontradas en peces teleósteos del Golfo de México y Vala et al. (1982) señalaron 10 especies y combinaciones nuevas en peces de Hawaii, del océano Atlántico, Índico, y del Mar Caribe. En el presente trabajo se describe una nueva especie de Haliotrema. 


\section{Materiales y Métodos}

Se capturaron nueve ejemplares de Lactophrys polygonia (Poey, 1876) en varias localidades de la Bahía

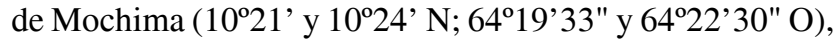
Estado Sucre, nororiente de la costa de Venezuela. Los ejemplares fueron trasladados vivos al Laboratorio de Parasitología de la Escuela de Ciencias de la Universidad de Oriente, en donde se procedió a extraérseles las branquias, éstas fueron colocadas en cápsulas de Petri con solución salina el $0,75 \%$ y se examinaron con ayuda de un microscopio estereoscópico. Los monogéneos hallados entre los filamentos branquiales fueron desprendidos con agujas de disección y retirados con goteros. Para la observación con miscroscopía del luz se procedió previamente a darles muerte por calor entre porta y cubre objetos, y en seguida fueron fijados con líquido de Bouin, deshidratados con etanol en concentraciones crecientes hasta $70 \%$, teñidos con acetocarmín de Semichon, deshidratados en etanol desde $70 \%$ hasta etanol absoluto, aclarados con aceite de clavo y montados permanentemente en bálsamo de Canadá. Los parásitos sometidos a microscopía electrónica de barrido (MEB) fueron fijados por lanzamiento en glutaraldehído, desecados por punto crítico utilizando un POLARON E-3000, metalizados con un Sputter Coater POLARON E5800 y visualizados mediante equipo SEM PHILIPS-XL30. Los dibujos fueron realizados con ayuda de una cámara clara adaptada a un microscopio de luz. Las medidas, realizadas sólo a ejemplares en preparaciones permanentes, fueron efectuadas con un ocular calibrado y son expresadas en micrones, indicando inicialmente el intervalo de la medida realizada en el eje longitudinal, a continuación la tomada en el eje transversal, seguidas del promedio entre paréntesis. Los parásitos hallados se contabilizaron para la determinación de la prevalencia, intensidad e intensidad media siguiendo lo señalado por Margolis et al. (1982) y la metodología descrita por Morales y Pino (1987).

\section{Descripción}

Haliotrema cumanensis sp. nov. (Figs. 1 - 3)

Descripción basada en 24 ejemplares maduros: 20 comprimidos y en preparaciones permanentes y cuatro preparados para MEB.

Cuerpo alargado, de 527-954 (677) por 109-209 (153), comprimido entre la región ovárica y orificio vaginal. Tegumento liso, al MEB se observan pequeñas papilas que se concentran principalmente en la región cefálica y en la zona media del cuerpo. Lóbulos cefálicos en número de cuatro, moderadamente desarrollados; tres pares de órganos cefálicos en forma de copa culminan en ellos. Dos pares de manchas oculares, ubicadas generalmente en la zona anterior a la faringe, constituidas por gránulos pequeños subesféricos. Opistohaptor fácilmente distinguible, de 63 a 142 (85) por 66 a 176 (131), conectado al resto del cuerpo por un corto pedúnculo, con una cavidad dorsal (observada mediante MEB) y provisto de dos pares de anclas disímiles, dos barras

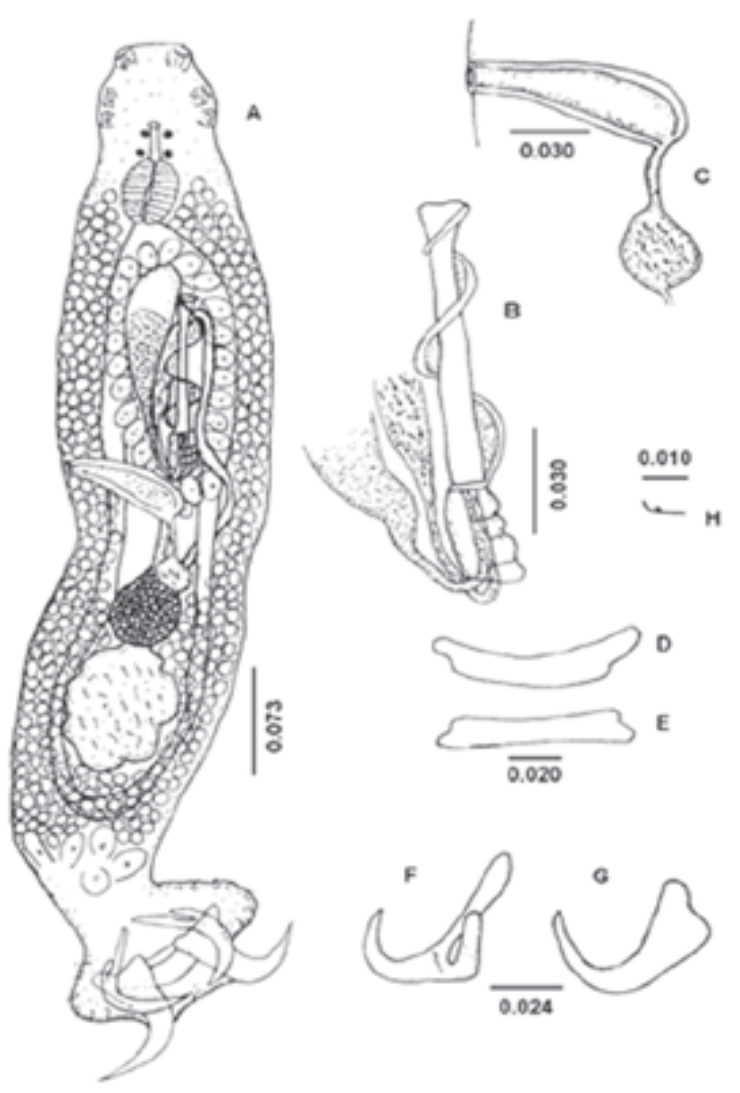

Figura 1. Haliotrema cumanensis n. sp. A. Holotipo, verme entero (vista ventral); B. Porción terminal del aparato reproductor masculino de paratipo (vista ventral); C. Vagina y receptáculo seminal de paratipo (vista ventral); D. Barra ventral de paratipo; E. Barra dorsal de paratipo; F. Ancla ventral de paratipo, G. Ancla dorsal de paratipo y H. Gancho larvario de paratipo.

y 14 ganchos larvarios alargados y en forma de «f», de 10 a 15 (12) de longitud. Anclas dorsales de 61 a 78 (68), con la raíz interna más larga y delgada que la superficial. Las anclas ventrales son más grandes que las dorsales, de 66 a 85 (75) de longitud, presentan dos raíces en forma de protuberancias romas, siendo la interna ligeramente más larga que la superficial. Barra dorsal, de 54 a 73 (67), con la porción media recta y provista de una protuberancia cerca de cada extremo. Barra ventral arqueada, más larga que la dorsal, de 73 a 93 (81), con una protuberancia cerca a cada extremo. Células caudales saculares presentes en la porción posterior del cuerpo. Boca subterminal separada de la faringe por un corto conducto. Faringe muscular, en forma de barril, de 34 a 51 (41) por 27 a 44 (37). Esófago tubular, de 10 a 17 (14), éste se bifurca en dos ciegos intestinales que se unen en la zona postesticular. Testículo postecuatorial, de bordes lobulados, en la zona postesticular. Testículo postecuatorial, de bordes lobulados, de 76 a 163 (116) por 49 a 127 (87). El vaso deferente emerge del borde anterior del testículo, desviándose para enlazarse con el ciego izquierdo, luego sigue su trayectoria ascendente hasta sufrir una acusada curvatura hacia abajo, ensanchándose para formar la vesícula seminal, que se ubica entre el reservorio prostático y el órgano copulador y se conecta a este último 


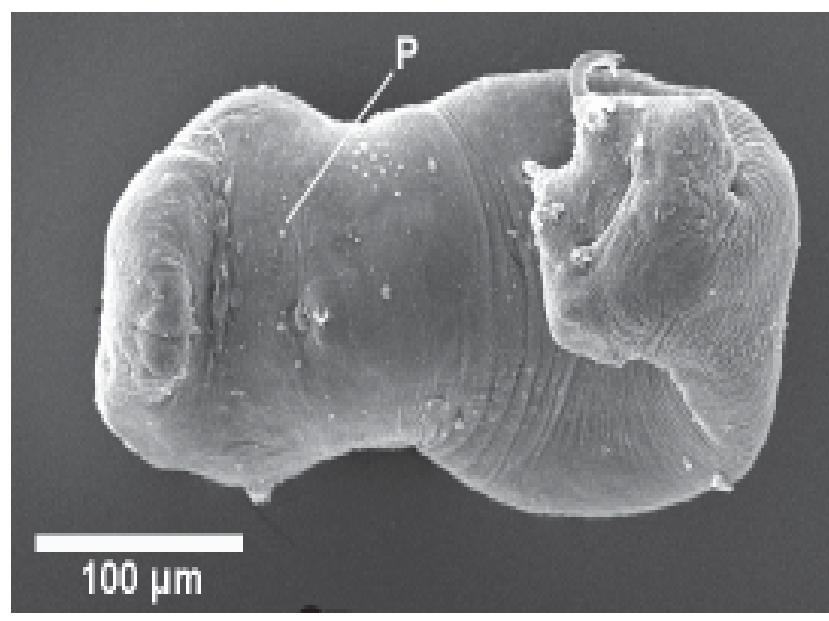

Figura 2. Haliotrema cumanensis $\mathrm{n}$. $\mathrm{sp}$. Microfotografía de barrido electrónico de verme entero, en donde se observan las papilas (p) del tegumento en la región media del cuerpo (vista ventral).

por la base. Complejo copulador constituido por un órgano copulador tubular, de 78 a 112 (98) por 7 a 15 (11), bipartido, con la fracción distal muy alargada y con una pieza accesoria membranosa en forma de vela, con el margen exterior engrosado, que la recorre en espiral en toda su extensión. Reservorio prostático sacular, de 54 a 111 (82) por 22 a 32 (26), diestro, del extremo posterior parte un delgado conducto que penetra en la base del órgano copulador. Células glandulares prostáticas grandes, distribuidas desde el nivel vaginal hasta la zona inmediatamente postbifurcal, de estas células parten dos conductos que penetran en el extremo anterior del reservorio prostático. Poro genital postbifurcal, a nivel medio del reservorio prostático y ligeramente sinistral. Ovario redondeado a ovoide, de 49 a 90 (63) por 27 a 71 (44), pretesticular. Útero corto y recto, éste se prolonga dorsalmente al órgano copulador hasta alcanzar al poro genital. Vagina sacular, de 61 a 98 (80) por 17 a 32 (25), ubicada en forma oblicua en la zona inmediatamente preecuatorial, abriendo hacia el margen derecho del cuerpo; de su margen lateroposterior parte un conducto corto y delgado que comunica con el receptáculo seminal pequeño y redondeado ubicado anterolateral al ovario. Glándulas de vitelo extendidas extra y supracecalmente, desde el nivel medio faringeal hasta cerca del extremo posterior del cuerpo, sin penetrar en el pedúnculo del opistohaptor. Huevos no observados.

\section{Resumen taxonómico}

Hospedero: Lactophrys polygonia (Ostraciidae); nombre local: «Torito».

Localización: filamentos branquiales.

Holotipo: depositado en el Museo Oceanológico «Hermano Benigno Román», Fundación La Salle, Campus de Margarita, Estado Nueva Esparta, Venezuela con el número de catálogo: EDIMAR-I-0814.

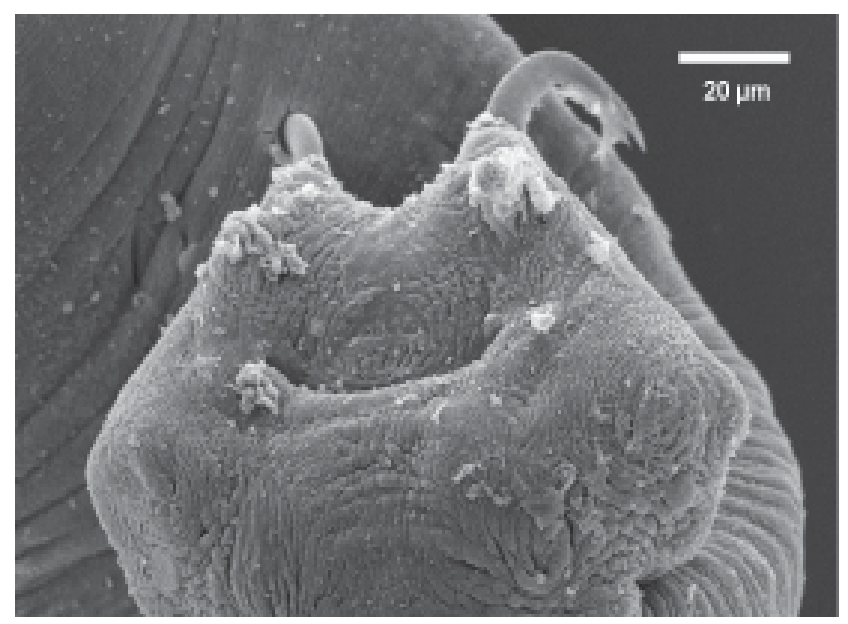

Figura 3. Haliotrema cumanensis n. sp. Microfotografía de barrido electrónico de la porción posterior del cuerpo en donde se observa la cavidad dorsal del opistohaptor (vista dorsal).

Paratipos: misma colección, número de catálogo: EDIMAR-I-0815; Colección Nacional de Helmintos, Instituto de Biología, Universidad Nacional Autónoma de México, número de catálogo: CNHE 5251 y en la colección personal del primer autor.

Intensidaad: $70-100$

Intensidad media: 85.8

Prevalencia: $77.7 \%$

Etimología: El nombre específico corresponde con la ciudad de Cumaná, capital del estado Sucre, donde se ubica la Bahía de Mochima, lugar de captura de los ejemplares.

\section{Comentarios taxonómicos}

El género Haliotrema fue creado inicialmente por Johnston y Tiegs (1922) para incluir la especie $H$. australe, hallada en Upeneus signatus, Queensland, Australia. Posteriormente Yamaguti (1963) realizó un estudio del holotipo de $H$. australe, depositado en el Museo Australiano en Sydney y encontró algunas discrepancias con la descripción original realizada por Johnston y Tiegs (1922), quienes no señalaron la unión de los ciegos intestinales y vaso deferente rodeando al ciego intestinal izquierdo, esto permitió diferenciar fácilmente Haliotrema de Ancyrocephalus Creplin, 1839, Parahaliotrema Mizelle y Price, 1964, Pseudohaliotrema Yamaguti, 1953; Pseudohaliotrematoides (Yamaguti, 1953) y Tetrancistrum Goto y Kikuchi, 1917. De estas especies, sólo ocho se han señalado como parásitos de ostrácidos; seis fueron descritas por Vala et al. (1982) para el Caribe: H. guadeloupensis y $H$. glandulosum, ambas halladas en Lactophrys triqueter y L. bicaudalis; H. kritskyi (Mizelle y Kritsky, 1969) en Acanthostracion polygonius; H. lactophrys (MacCallum, 1915) en A. quadricornis y A. polygonius; H. minutum en A. polygonius y H. torridum en L. triquete y A. polygonius. Estas especies comparten con $H$. cumanensis la presencia de una pieza accesoria veliforme que recorre en espiral la 
parte distal del órgano copulador; no obstante, $H$. cumanensis se diferencia de éstas por presentar la parte distal del órgano copulador claramente tubular y recto (no cónico y ligeramente curvo), por poseer un testículo de bordes lobulados y por presentar dos procesos basales largos y bien diferenciados en las anclas ventrales. Las dos especies restantes, $H$. trochaderoi y $H$. crymanum, fueron descritas por Klassen (1993) parasitando peces del género Ostracion en el Pacífico e Indo-Pacífico. Haliotrema cumanensis puede diferenciarse de estas dos especies por la ausencia de la pieza accesoria veliforme que recorre en espiral la parte distal del órgano copulador, además de las características que la diferencian de las otras seis especies que parasitan a ostrácidos.

Existen otras especies que no han sido registradas en ostrácidos y que comparten con $H$. cumanensis la presencia y morfología de la pieza accesoria del órgano copulador, tal es el caso de $H$. spiralis Yamaguti, 1968, H. australe Johnston y Tiegs, 1922; $H$. allornata Paperna, 1972; $H$. caballeroi Euzet y Vala, 1977; H. caraibensis Euzet y Vala, 1977; H. chrysostomi Young, 1968; H. dascyllussi Paperna, 1972; H. epinepheli Young, 1968; H. macassariensis Yamaguti, 1963; H. cornutus (Mizelle y Kritsky, 1969) Vala, Maillard y Overstreet, 1982 y H. pacificus (Mizelle y Kritsky, 1969) Vala, Maillard y Overstreet, 1982; sin embargo, la forma del conducto copulador tubular, acentuadamente alargado, no en forma de cuerno, y la condición lobulada del testículo permiten, entre otras características particulares, distinguirlas de H. cumanensis.

\section{Literatura citada}

Bychowsky, B. E. y L. F. Nagibina. 1970. On the new and little known species of the genus Haliotrema Johnston and Tiegs 1922 (Monogenoides). Zoologicheskii Zhurnal 49: $1789-1801$.

Johnston, T. H. y O. W. Tiegs. 1922. New gyrodactylid trematodes from Australian fishes, together with a reclassification of the superfamily Gyrodactyloidea. Proceedings of the Linnean Society of New South Wales 47: 83 -131.

Klassen, G. J. 1993. Haliotrema species (Monogenea: Ancyrocephalidae) from Indo-Pacific boxfishes (Tetraodontiformes: Ostraciidae). Canadian Journal of Zoology 71: 2099- 2110.

Kritsky, D. C. y F. Stephans. 2001. Haliotrema abaddon sp. n. (Monogenoidea: Dactylogyridae) from the gills of wild and marinicultured West Australian dhufish,
Glaucosoma hebraicum (Teleostei: Glaucosomatidae), in Australia. Journal of Parasitology 87: 749-754.

Kritsky, D. C. y W. A. Boeger. 2002. Neotropical monogenoidea. 41: New and previously described species of Dactylogyridae (Plathyhelminthes) from the gills of marine and freshwater perciform fishes (Teleostei) with Zoosystema 24: 7-41.

Margolis, L., G. Esch, J. Holmes, A. Kuris y G. Schad. 1982. The use of ecological terms in parasitology (report of an ad hoc committee of the American Society of Parasitologists). Journal of Parasitology 68: 131- 133.

Morales, G. y L. Pino. 1987. Parasitología cuantitativa. Fundación Fondo editorial Acta Científica Venezolana. Caracas, Venezuela. 132 p.

Vala, J. C., C. Maillard y R. Overstreet. 1982. Haliotrema (Monogenea: Ancyrocephalinae) from ostraciid fish in Guadalupe, West Indies. Journal of Parasitology 68: $1130-1137$.

Yamaguti, S. 1963. Systema helminthum. Volumen IV: Monogenea and Aspidocotylea. Interscience Publishers, New York. 699 p.

Yamaguti, S. 1968. Monogenetic trematodes of Hawaiian fishes. Keigaku, Tokio. 436 p.

Young, P. C. 1968. Ten new species oh Haliotrema Johnston and Tiegs, 1922 (Monogenoidea: Dactylogyridae) from Australian fishes and a revision of the genus. Journal of Zoology, London 154: 41-75.

Zhukov, E. V. 1976. New monogenean species of the genus Haliotrema Johnston and Tiegs, 1922 from the Gulf of Mexico Fishes of the family Lutjanidae. In Fauna, systematic and phylogeny of Monogenoidea. Proceedings of the Institute of Biology and Pedology of the Far-East. Science Centre of the Academy of Sciences of the USSR., New Series 35: $33-47$.

Zhukov, E. V. 1980a. New species of monogeneans from genus Haliotrema from the gills of fish from families Chaetodonthidae and Scaridae in the Gulf of Mexico. Parazitologiya 14: $308-313$.

Zhukov, E. V. 1980b. Monogenea of the genus Haliotrema Johnston et Tiegs, 1922, from the gills of fishes (Holocentridae and Acanthuridae) of the Gulf of Mexico. Parazitologichezkii Sbornik 29: 41 - 52.

Zhukov, E. V. 1981. New monogenean species of the genus genus Haliotrema Johnston et Tiegs, 1922 from the gills of fishes Pomadasyidae and Mullidae from the Gulf of Mexico. Parazitologichezkii Sbornik 30: 179 -189. 